This is the author's version of the work. It is posted here for personal use, not for redistribution. The definitive version was published in Health Information \& Libraries Journal 2014;31:13347. DOI: $10.1111 /$ hir. 12062

\title{
Choosing and using methodological search filters: searchers' views
}

Sophie Beale, ${ }^{1}$ Steven Duffy, ${ }^{2}$ Julie Glanville, ${ }^{3}$ Carol Lefebvre, ${ }^{4}$ Dianne Wright, ${ }^{3}$ Rachael McCool, ${ }^{3}$ Danielle Varley, ${ }^{3}$ Charles Boachie, ${ }^{5}$ Cynthia Fraser, ${ }^{5}$ Jenny Harbour, ${ }^{6}$ Lynne Smith ${ }^{6}$

1. Liverpool Reviews and Implementation Group, University of Liverpool, Liverpool, UK.

2. Centre for Reviews and Dissemination, University of York, York, UK.

3. York Health Economics Consortium (YHEC), University of York, York, UK.

4. Lefebvre Associates Ltd, Oxford, UK.

5. Health Services Research Unit, University of Aberdeen, Aberdeen, UK.

6. Healthcare Improvement Scotland, Glasgow, UK.

\section{Abstract}

This paper reports on findings from interviews and a questionnaire survey that were conducted as part of a larger UK Medical Research Council-funded study to help understand more about how librarians and researchers choose, and use, methodological search filters.

The 16 interviewees comprised information specialists working in, or for, the National Institute for Health and Care Excellence (NICE). An online questionnaire was used to collect the views of the wider (national and international) systematic review, health technology assessment, and guidelines development community. Potential respondents participated in the questionnaire via notices on relevant email discussion lists, posts on the York Health Economics Consortium (YHEC) Facebook page, and messages sent out via the YHEC Twitter account. Ninety questionnaires were completed.

This study found that the use of search filters tends to be linked to reducing large literatures, introducing focus, and assisting with searches which are based on a single study type. Questionnaire respondents use numerous ways to identify search filters and can find choosing between different filters problematic because of knowledge gaps and lack of time. Factors they would find helpful include making information about filters less technical, offering filter ratings, and providing more detail about filter validation strategies and filter provenance. 


\section{Corresponding author:}

Julie Glanville,

York Health Economics Consortium (YHEC),

University of York,

York, YO10 5NH, UK.

Tel: 01904324832

\section{Keywords}

Databases, filter performance, information specialists, librarians, methodological search filters surveys

\section{Key messages:}

- $\quad$ Information specialists surveyed develop new search strategies at least once a week.

- $\quad$ Nearly all information specialists surveyed use methodological search filters.

- $\quad$ The majority of information specialists surveyed use different filters depending on whether their search requires sensitivity or precision.

- $\quad$ Half of information specialists surveyed sometimes amend published search filters.

- Information on the sensitivity and precision of a search filter is an important factor for selection of an appropriate filter.

\section{Introduction}

Search filters, also known as 'hedges' or optimal search strategies, have been developed to assist information specialists and librarians, researchers, clinicians and other searchers to retrieve different types of evidence from bibliographic databases. They consist of combinations of search terms, generally drawing on both free-text and controlled vocabulary (such as Medical Subject Headings in Medline). ${ }^{1}$ They are typically combined with sets of subject terms. ${ }^{2-5}$

Search filters are an important tool for those who are searching for specific research designs or subjects because they can save time in identifying some of the elements of the strategy and, where search filters have been validated, can provide tools whose reliability can be referenced. ${ }^{3 \text {, }}$

4 Search filters are a tool of particular relevance to searchers and researchers producing systematic reviews and clinical guidelines, since they can provide some standardization in a field where standards and guidance are sparse and sensitive retrieval is important. 
In 2004, a telephone and fax survey to determine the awareness and use of methodological search filters amongst health and scientific librarians in the UK reported a high level of awareness but a low level of usage. ${ }^{2}$ Since 2004 many more filters have been published. ${ }^{3,4}$ Filters are now incorporated into database interfaces, such as the PubMed Clinical Queries ${ }^{4}$, as well as being reported in medical and scientific journals. ${ }^{3}$

Research methods to develop search filters include the identification of gold standards to derive and test strategies, the use of test and validation sets of records to test the performance of filters, the use of statistical techniques to identify search terms and to test various search filters to identify those which perform best in terms of sensitivity and precision. ${ }^{2}$ The methodology of search filter development is evolving. ${ }^{6}$ The use of research methods to develop filters is improving the probability that search filters will be reliable and consistent in their performance when used with a range of subject strategies. ${ }^{5,7}$

As search filters have increased in number, critical appraisal tools have been developed which can be used to assess or appraise search filters. ${ }^{2,10,11}$ These critical appraisal tools can help in the selection of search filters for specific tasks by identifying those filters which have been developed using methods which are liable to minimize bias and which have been tested in terms of their ability to retrieve relevant records with the minimal proportion of irrelevant records. Searchers and researchers need to be aware of the process and limitations involved in search filter development so that they are able to make informed decisions about whether to use them.

In 2011 the Medical Research Council (MRC), in partnership with the (now) National Institute for Health and Care Excellence (NICE) announced a programme of funding for methodological research to underpin NICE decision making. Given the importance of evidence identification for the work of NICE in producing guidance and guidelines, and the general desire for standardization in research approaches, research into filter use, we developed a proposal to explore filter use. The objectives of this research project were to improve our understanding of filter use by searchers and researchers, how searchers and researchers choose search filters and what information they would like to receive to inform their choices. The proposal was funded as MRC research grant G0901496 ${ }^{12}$ (May 2010 to May 2012) and the research was undertaken by information specialists and researchers from the York Health Economics Consortium at the University of York, the UK Cochrane Centre (now based at Lefebvre Associates Ltd), the University of Aberdeen, and Healthcare Improvement Scotland.

The research involved a multi method approach:

- $\quad$ Five literature reviews were carried out to investigate different aspects of performance measurement in search filters and diagnostic test accuracy studies (to which they are analogous), their reporting and the selection of search filters by searchers and researchers

- Interviews were carried out and a web-based questionnaire was made available to gain information on current filter use 
- $\quad$ The reviews, interviews and questionnaire survey then informed the development of pilot filter performance visualisations and guidance on gathering and reporting search filter performance.

This paper reports the findings from the interviews and questionnaire survey which were conducted to improve the project team's understanding of how searchers and researchers choose and use search filters and also to understand what information searchers and researchers would like to receive to inform their choice of search filters.

\section{Methods}

\section{Study design}

\section{Phase I: Semi-structured interviews}

The funding for this research was awarded as part of a programme to benefit NICE working practices. In the light of that key audience for this research, the views of NICE information specialists and researchers seemed especially important for the project. Information specialists and Project Managers working for the National Institute for Health and Care Excellence (NICE), the NICE collaborating centres and the NICE Evidence Review Groups (ERGs) were invited to take part in a telephone interview. Each interview was recorded and took no more than 45 minutes. After each interview, an email containing a summary of the key points raised during the interview was sent to each interviewee. The interviewee was offered the opportunity to check the notes for accuracy and to add any additional points that might have occurred to them after the interview had ended.

\section{Phase II: Questionnaire survey}

A questionnaire survey was developed to obtain information on a wider community of searchers' knowledge of and use of search filters. This was intended to benefit NICE through providing information on how other searchers might use, choose and amend search filters. The questions in the survey were based on the findings from the reviews and the interviews which had already been undertaken as part of this project. A web-based questionnaire comprising 23 questions was developed (Appendix).

The questionnaire was made available on the YHEC website (www.yhec.co.uk). NICE information specialists and NICE Project Managers were invited to complete the questionnaire and it was also used to collect the views of the wider (national and international) systematic review, health technology assessment, and guidelines information community via invitations sent out on seven email lists:

- Lis-medical (1,523 individuals belonging to an open discussion list for members of the UK medical \& health care library community and other interested information workers); 
- $\quad$ irmg@lists.cochrane.org (204 subscribers belonging to the open discussion list of the Cochrane Information Retrieval Methods Group)

- $\quad$ issg@lists.shef.ac.uk (subscribers are information specialists who work for the evidence review groups providing services to NICE);

- $\quad$ isg-informationresources@htai.org (subscribers are information specialists who are members of the HTAi organisation);

- Campbell IRMG (30 subscribers belonging to the Campbell Information Retrieval Methods Group);

- Cochrane TSCs (100 members of the Cochrane Trials Search Coordinators email discussion list);

- $\quad$ EAHIL-L@MAILTALK.AC.UK (1,000 members of the discussion list of the European Association for Health Information and Libraries).

Potential respondents were also alerted to the questionnaire via posts on the YHEC Facebook page, and messages sent out via the YHEC Twitter account. The survey was available for completion during a four week period (22 July 2011 - 18 August 2011), with email reminders being sent out one week before the final deadline to maximize the response rate.

\section{Results}

\section{Data collection}

Twelve interviews were conducted in May and June 2011. Ten of the interviews involved one interviewee, one interview involved two interviewees and another interview involved four interviewees. In total 16 people took part in the interviews. Fifteen of the interviews were carried out by telephone whilst one was carried out face-to-face.

A total of 90 questionnaires were completed. It is not possible to calculate a response rate as individuals may have been members of more than one discussion list. Furthermore, the number of respondents who were alerted to the questionnaire via the Twitter or Facebook messages is unknown. Just over three-quarters of respondents $(70 / 90 ; 77.8 \%)$ worked directly in information or library services, whist the remaining respondents came from a range of backgrounds and included a consultant physician, an associate scientist, a medical documentalist and a learning resources officer. The questionnaire was completed by experienced searchers, with nearly half $(44 / 90 ; 48.9 \%)$ having 11 or more years of database searching experience. Additionally, threequarters of questionnaire respondents $(68 / 90 ; 75.6 \%)$ reported that they developed searches at least once a week, half of whom indicated that they developed searches daily.

All but one respondent used MEDLINE, and most (84/90; 93.3\%) used The Cochrane Library databases. About three quarters used EMBASE (70/90; 77.8\%) and CINAHL (67/90; 74.4\%), whilst a little under two-thirds (56/90; 62.2\%) used PsycINFO. 


\section{When do researchers use search filters?}

\section{Interviewees}

Findings from the interviews suggested that search filters are only rarely used by NICE information specialists. The reasons given varied according to the NICE output and are as follows:

\section{- $\quad$ Clinical guidelines}

For short clinical guidelines the team only use search filters on the rare occasions when the search is restricted to a particular study design

- Diagnostic guidance

The filters available do not work well

- Interventional procedures guidance

Searches are carried out at the point in time when (or before) products get a Conformité Européenne (CE) mark, a symbol given to products that conform with relevant EU health and safety directives, and tend to be internet based as, at this stage, there are few publications and few publications in databases

- Technology appraisals

- Single Technology Appraisals involve a review of the literature reviews submitted to NICE by the manufacturer and the ERG's task is only to develop searches to test the validity of the searches carried out by the manufacturer

- Multiple Technology Appraisals are population, intervention, comparison and outcome (PICO) driven and do not require methods filters.

Interviewees reported, however, that they sometimes used search filters under the following circumstances:

- When carrying out searches for a particular study type

- To help focus the question in order to achieve a manageable numbers of records

- On searches for small projects with limited resources

- To identify economic evidence.

\section{Questionnaire respondents}

Eighty-five of $90(94.4 \%)$ respondents indicated that they had used methodological search filters, and 5/90 (5.6\%) indicated that they had not (question 6).

About three-quarters of respondents said they use search filters for extensive searches to inform guidelines or systematic reviews (question 7), whilst just over half said they would use them for rapid searches to answer brief questions and a similar number would use them for scoping searches to estimate the size of the literature on a topic (Table 1). 
Other reasons that were given for using search filters included:

- $\quad$ To reduce results to a manageable size/narrow down results

- $\quad$ To begin to identify MeSH and text words to use in developing a strategy

- If the customised limits provided by the databases cannot be relied upon

- Monitor trends.

Table 1 Circumstances in which questionnaire respondents use search filters (question 7).

\begin{tabular}{|l|c|c|}
\hline Circumstances in which search filters are used & $\begin{array}{c}\text { Number of } \\
\text { respondents }\end{array}$ & Percentage \\
\hline Extensive searches to inform guidelines or systematic reviews & 69 & $76.7 \%$ \\
\hline Rapid searches to answer brief questions & 55 & $61.1 \%$ \\
\hline Scoping searches to estimate the size of the literature on a topic & 53 & $58.9 \%$ \\
\hline None of the three reasons above & 7 & $7.7 \%$ \\
\hline Other reasons & 12 & $13.3 \%$ \\
\hline
\end{tabular}

Benefits of using filters (question 19) included that filters help focus results (38/90; 42.2\%), are tried and tested $(17 / 90 ; 18.9 \%)$, save time $(9 / 90 ; 10 \%)$, and offer transparency and consistency $(5 / 90 ; 5.6 \%)$. Other benefits cited by individuals include helping to estimate workload in project planning and offering a "conceptual mapping of thoughts".

Limitations of using filters (question 20) were identified by respondents as the possibility of missing relevant studies $(34 / 90 ; 37.8 \%)$, that filters might not always be fit for purpose (20/90; $22.2 \%)$, there may be difficulties in appraising the filter $(9 / 90 ; 10 \%)$ and that filters are only as good as their developer or the database indexing (13/90; 14.4\%. Other limitations cited by single respondents were that filters may produce too many results, there is a lack of instructions on how to cite the filters, it can sometimes be difficult to choose between filters, and filters are sometimes chosen as a compromise.

\section{How do searchers identify search filters?}

\section{Interviewees}

The most frequently reported methods used by interviewees to identify search filters were:

- $\quad$ The InterTASC information specialists' Sub-Group (ISSG) Search Filters Resource ${ }^{3}$

- $\quad$ Posting messages to discussion lists 
- $\quad$ Consulting the Cochrane Handbook

- Discussion with colleagues

- $\quad$ Using an in-house search manual

- $\quad$ Reviewing previous project methods.

\section{Questionnaire respondents}

Respondents searched a range of sources to identify search filters (question 10). The most frequently reported sources for study design filters were those produced by the McMaster Hedges Team. These filters are also included in many interfaces to MEDLINE. Further details of the seven most reported resources are provided in Table 2.

Table 2 Search filter sources reported by questionnaire respondents.

\begin{tabular}{|c|c|c|c|c|c|c|}
\hline \multirow[b]{2}{*}{ Resource } & \multicolumn{6}{|c|}{ Study Design } \\
\hline & RCTs & $\begin{array}{l}\text { Systematic } \\
\text { reviews }\end{array}$ & DTAs & Prognosis & Etiology & $\begin{array}{l}\text { Other } \\
\text { trials }\end{array}$ \\
\hline $\begin{array}{l}\text { Cochrane (this term is used to } \\
\text { capture references to Cochrane } \\
\text { reviews, the Cochrane } \\
\text { Handbook, the Cochrane } \\
\text { Collaboration (not otherwise } \\
\text { specified)). }\end{array}$ & 33 & 5 & 1 & 1 & 1 & 2 \\
\hline $\mathrm{CRD} / \mathrm{DARE}^{13}$ & 2 & 4 & 1 & 1 & 1 & 1 \\
\hline $\begin{array}{l}\text { InterTASC ISSG Search Filter } \\
\text { Resource }^{3}\end{array}$ & 4 & 2 & 2 & 2 & 2 & 1 \\
\hline McMaster Hedges Team $^{14}$ & 13 & 13 & 11 & 16 & 13 & 2 \\
\hline $\begin{array}{l}\text { Respondent's own filter/own } \\
\text { adaptation of a filter }\end{array}$ & 7 & 6 & 5 & 5 & 3 & 9 \\
\hline $\begin{array}{l}\text { Search filters built into } \\
\text { databases } \\
\text { Ovid/PubMed/MEDLINE/NHS } \\
\text { Athens }{ }^{15-17}\end{array}$ & 16 & 23 & 10 & 12 & 13 & 4 \\
\hline $\operatorname{SIGN}^{18}$ & 8 & 11 & 5 & 1 & 1 & 1 \\
\hline
\end{tabular}

When describing which filters they currently use (question 11), respondents cited search filters for RCTs $(67 / 90 ; 74.4 \%)$ and systematic reviews $(61 / 90 ; 67.8 \%)$ most frequently. The most frequently cited filters in a specific topic were the Cochrane filters to identify RCTs (33/90; $36.6 \%)$.

When asked how they keep up to date with search filters (Question 15) the majority of respondents $(60 / 90 ; 33.33 \%)$ indicated that they read journal articles, $33.33 \%$ also used 
information provided by managers or a work colleague, 50/90 (55.55\%) used websites, 67/90 (74.44\%) used professional development meetings and training events, 57/90 (63.33\%) used email lists and 24/90 (26.66\%) used current awareness services. Email lists cited included canmed-lib, clinlib, cochrane information retrieval methods group, cochrane trials search coordinators, eahil-l, evidence-based health, evidence-based-libraries@jiscmail.ac.uk, expertsearching, hslg, intertasc information specialists subgroup, isg-informationresources, lib-helix, lis-medical, lis-nursing, local health libraries network, medlib, ncc-informationspecialists, sys-review@jiscmail.ac.uk, webenz.

\section{How do researchers decide which search filters to use?}

\section{Interviewees}

Sensitivity and precision were important to most interviewees. Several interviewees indicated that they would test the filters against a target set of references to see which filter identified most references, although the approaches described tended to be pragmatic in nature. Other approaches included:

- Using the most up to date filter

- $\quad$ Considering the credentials of the people/organization that developed the filter

- $\quad$ Assessing the methods used to develop the filter

- $\quad$ Reviewing details about the filter published in the academic literature

- $\quad$ Taking advice from senior colleagues.

\section{Questionnaire respondents}

When asked about how they typically use search filters, about four-fifths of respondents (73/90; $81.8 \%)$ indicated that they use different filters depending on whether their search needs to be sensitive or precise (question 9).

Respondents were asked how they decide which filter to use by selecting one, or more, options from a list (question 12). Just over half of the respondents reported that they generally use the filters that are already available in the database being searched (48/90; 53.3\%) ( 
Table 3), rather than typing in another filter. 
Table 3 How do you decide which filter to use?

\begin{tabular}{|l|c|c|}
\hline Typical practice & Number & Percentage \\
\hline I research the available filters and choose the best for my purposes & 51 & $56.7 \%$ \\
\hline $\begin{array}{l}\text { I use the filters available in the database interfaces I use, e.g. Clinical } \\
\text { Queries }\end{array}$ & 48 & $53.3 \%$ \\
\hline Custom and practice - I've always used the same filters & 34 & $37.8 \%$ \\
\hline Guidance from a colleague & 34 & $37.8 \%$ \\
\hline $\begin{array}{l}\text { I follow standard operating procedures/guidance on filters provided by } \\
\text { my organization }\end{array}$ & 22 & $24.4 \%$ \\
\hline I use international/national guidance on best practice & 21 & $23.3 \%$ \\
\hline
\end{tabular}

Other approaches used by respondents to help them decide which filters to use were trial and error, or comparing results and comparing filters' sensitivity and precision.

Respondents were asked how, when faced with a choice of methodological filters, they chose which one to use (question 21). Fifteen respondents $(15 / 90 ; 16.7 \%)$ said that they required a description of the filter, $25(25 / 90 ; 27.8 \%)$ required information on the performance of the filter in terms of validation, while $18(18 / 90 ; 20 \%)$ required information on sensitivity and precision. Ten respondents $(10 / 90 ; 11.1 \%)$ required information on who had published the filter and six said they would ask colleagues for advice.

When asked about information which had helped with filter selection in the past (question 16) similar responses were received to question 21 but in addition single respondents recorded the following observations:

- $\quad$ Personal knowledge or choosing the one that looks logical

- $\quad$ Length - 'the shorter the better'

- Focus

- $\quad$ Flexibility/modifiability

- $\quad$ Testing against a target reference list

- InterTASC ISSG website

- Assessing the search terms within the strategy.

Once they had found a search filter, just over half of the respondents $(50 / 90 ; 55.6 \%)$ reported that they sometimes amend search filters (apart from adding a subject search), whilst four respondents $(4 / 90 ; 4.4 \%)$ indicated that they always amend search filters, a third (30/90; 33.3\%) do not amend them (question 13), whilst six individuals did not respond to this question. Twenty- 
six respondents $(26 / 90 ; 28.9 \%)$ indicated that they amended filters to improve sensitivity and/or specificity (question 14), and thirty respondents (30/90; 33.3\%) indicated that they amended search filters by adding or removing subject headings, text words or both. Other methods used to amend search filters mentioned by respondents included:

- $\quad$ Adapting the filter to another database $(n=1)$

- Looking at adjacency or truncation $(n=6)$

- $\quad$ Examining which lines of syntax are producing zero, or too many results $(n=1)$

- Weighting word algorithms $(\mathrm{n}=1)$

- $\quad$ Taking advice from other information specialists $(n=1)$

Nearly all of those respondents who amended search filters $(45 / 54 ; 83.3 \%)$ tested the effect of the amendment (question 14) by either comparing the results with and without the filter, or by whether known relevant papers had been identified. Three-quarters of respondents (68/90; $75.9 \%$ ) documented their amendments when they wrote up the searches, using diverse approaches including reproducing the entire search string with a written summary and rationale for changes and effects, keeping detailed spreadsheets and notes on strategy changes, and including narratives on filter amendments in the Methods section of the review.

\section{What information would help researchers choose between filters?}

\section{Interviewees}

Some interviewees expressed a preference for a simplified measure of search filter validation, such as a rating system. This was an expression of their lack of time to fully critically appraise the methodological robustness of filter design. One interviewee stressed that they would need to have confidence in whoever carried out the rating. Other interviewees said that a summary documenting sensitivity and precision would be useful, although it was recognized that this might be subjective.

\section{Questionnaire respondents}

In the 56 free-text answers supplied in response to question 21 respondents indicated that information on search filter performance measures such as validation (25/90; $27.8 \%)$, sensitivity and precision $(18 / 90 ; 20 \%)$ and a description of the filter $(15 / 90 ; 16.7 \%)$ had helped them in choosing which filter to use. Other information identified as useful for selecting a filter included:

- $\quad$ Results of own testing (11 respondents)

- $\quad$ Colleague recommendations/discussion (6 respondents)

- Knowledge that the filter is appropriate for a relevant database (4 respondents);

- $\quad$ Knowledge of the creator/developer (3 respondents);

- $\quad$ Simplicity/easy to understand (3 respondents); 
- $\quad$ Ease of use (including automatic loading) (2 respondents).

Respondents reported that the main factors which would make choosing a filter easier were the availability of a critical appraisal or evaluation (16/90; 17.7\%) and more information (15/90; $16.6 \%$ ), including the effectiveness of the filter, what it does or what it provides, what it excludes, its limitations, when it was last updated, advantages and disadvantages, sensitivity and precision, and what testing had been completed. Respondents also reported that they wanted to be confident in the author/developer $(10 / 90 ; 11.1 \%)$ and that having the filter available in a central storage location was an important consideration (7/90; 7.7\%).

Individuals also suggested that the following changes would make choosing between search filters easier:

- Improved labelling/indexing of articles so they might be more easily retrieved (1 respondent)

- $\quad$ More up to date coverage on the ISSG Search Filter Resource website (1 respondent)

- $\quad$ The need for more 'professional noise' about a new filter (1 respondent).

\section{Discussion}

In 2004, Jenkins and Johnson reported that, although researchers were aware of filters, there was a low level of usage. ${ }^{2}$ Since then, it appears that more people are using filters to inform their research, and filters are being used for a range of searching tasks.

The present study has several limitations. Although we do not know what proportion of search filter users we have reached, questionnaire analyses show that our sample includes librarians and other researchers involved in supporting systematic reviews, technology appraisals and guideline development, as well as healthcare librarians, all of whom represent our target audience. From the mailing lists of which respondents report being members we can tell that many are information specialists supporting the production of health technology assessments, guidelines and systematic reviews. We therefore expect the results to be broadly generalisable. The mailing lists we circulated had at least 2857 subscribers, which does however mean that the 90 respondents represent a low response rate. The mailing lists we used ranged from lists with high proportions of information specialists with roles similar to NICE information specialists to more general lists where many of the members might not routinely use search filters.

In terms of in depth interviews, our sample was limited to NICE staff, for whose benefit the research was being undertaken. It would be useful for future research to interview searchers and researchers from other settings, to understand whether the NICE experience is generalisable. 
The questionnaire we developed was quite lengthy and, in retrospect, might have benefited from being shorter. However, the response rate to early questions is similar to that of later questions, suggesting that the length of the questionnaire did not deterrent to any of the individuals who actually submitted a response. It might have helped to achieve more standardized results and fewer ambiguous answers if we had given respondents more multiple choice questions. Respondents described resources quite vaguely at times and sometimes the same resource was described using several different names. We have made some assumptions about the variant naming of widely used resources which we have applied to provide a more succinct report and to ensure that the most frequently reported resources are identified as such. We have not, however, corrected what may be 'errors' in the responses, for example where certain filters may be incorrectly described or ascribed to the wrong author or organization.

\section{When do searchers and researchers use search filters?}

The awareness and use of search filters seems to have developed considerably in the decade since Jenkins' paper. ${ }^{2}$ Most respondents seem to know where to look for filters from wellestablished producers and collections. However, the responses demonstrate a wide variation in the confidence with which questionnaire respondents choose filters. There are also contradictions between the difficulties respondents express in terms of selecting between filters (acknowledging the possible complexities of filter design) and the commonplace practice of searchers adapting published strategies to fit their own requirements (ignoring the fact that many filters are designed to perform in a quite specific way). Several respondents have developed their own filters for local use.

The responses indicate that search filters are used more frequently for large scale reviews and slightly less often for simpler scoping and rapid searches. This may reflect different practices in scoping and rapid searches because fewer resources will be searched and less sensitive subject searches will be employed because of the limited timescale. Adding a filter to an already focused search might be seen as risking missing studies. For all types of searches, search filters offer an opportunity to focus the numbers of records retrieved, which can be helpful when time is limited. Search filters are predominantly viewed by respondents as a tool to maximize sensitivity rather than precision (although this is not the intended objective of all filters), but seem to be used to achieve optimal sensitivity and precision.

\section{What information would help researchers choose between filters?}

The responses to the questionnaire have many messages for search filter designers. Filter performance measures need to be signposted more clearly and succinctly to help searchers make better use of available filters. Filter and websites designers should present less information (to avoid information overload) and to ensure that performance information can be clearly seen. Respondents also reported that they wanted to be confident in the author/developer. While the provenance of filters is clearly important to some searchers, there 
are no established parameters to measure this confidence. Clear authorship labelling and provision of detailed methods to show the robustness of the development methods would not only assist users of filters but would also help filter designers achieve recognition for their filters. The convenience of having filters by well-established producers available within database interfaces (such as the PubMed Clinical Queries) encourages their use. However, the most convenient search filters may not always be the best for the task and searchers and researchers need to know how to choose when a range of sensitive, precise or optimal strategies are offered.

Respondents require more information on the validation of search filters. They value and use resources such as the ISSG Search Filter Resource and the filters of the McMaster Hedges Team. The former provides a listing of filters in one place by study design, which has a convenience factor. The latter provide search filters developed with documented methods within database interfaces and are 'badged' with the authority of both the research team and the National Library of Medicine. In contrast to the methodological and publisher quality seals of the McMaster filters, the BMJ Clinical Evidence and SIGN contain little information on filter production and/or validation. However, the filters on these websites seem to be widely used, suggesting that the authorship is the seal of quality.

Respondents did not necessarily feel that all their requirements were currently being met. They would like translations of filters for different databases and interfaces, more strategies independent of indexing language (to facilitate transferral across databases) and filters for a wider range of study designs and other topics. This provides a research agenda for any search filter authors willing to take up the challenge.

Respondents keep informed about developments in search filters through a wide variety of methods and resources, which suggests that search filter and website designers face a marketing challenge. Highlighting new filters to key audiences such as information specialists and systematic reviewers by inclusion in resources such as the Cochrane Handbook and the ISSG Search Filter Resource would help to promote new filters beyond the simple publication of a journal paper. In addition a large number of email lists are used for current awareness and promotion of new filters through those lists would seem an efficient way to reach potential users.

Although the use of search filters seems to be quite widely documented, and amendments noted in search reports, there seems to be scope for promoting clarity around the use, and amendment, of search filters. This, again, is an issue for filter authors and website producers. There is clearly a large amount of ad hoc filter amendment being undertaken: searchers take filters and adapt them for their own purposes. This would seem to indicate a lack of awareness that the filters may be designed for a purpose, or arrived at after extensive exploration to justify the use of specific terms. The performance assessment of amended search filters does not seem to be a priority for many searchers. Filter developers should consider how they want their filters to be used and perhaps attach guidance or caveats to the filters. Guidance for filter adaptation may also be merited so that filter developers are credited for the original work but 
absolved from the effects of the adaptations. Many filter developers retain their gold standards and might be willing to test adaptations.

The original impetus for many search filters was to maximize sensitivity, but increasingly, possibly due to limited resources, searchers seem to be demanding improvements in precision. Future filter developments (for interfaces which use Boolean searching) need to continue to improve precision. The advent of full text searching and semantic analysis of both full text and bibliographic records may see filters used in different ways in future. For example, sensitive filters might be used to identify records from databases. These results might then be processed using semantic analysis software trained to identify records of specific types. The latter approach will have search algorithms (filters) which are more like semantic rules than the dichotomous (relevant/not relevant) search filters we see in bibliographic databases such as

MEDLINE. Recent search filters have used textual analysis approaches in their design. ${ }^{6,19}$ The extent to which textual analysis alone can be relied upon in the future to distinguish relevant records from irrelevant records is under investigation. ${ }^{19,} 20$ When using semantic analysis approaches the onus will be on the searcher to select the performance levels, that is, an acceptable probability of a record being relevant is acceptable.

\section{Future research}

Areas for future research could include presenting search filter performance in different ways and testing searchers' understanding of the filter performance trade-offs offered. It would also be helpful to gain more information on exactly how search filters are amended in practice, in order to inform filter design: filter designers assume searchers want sensitive filters, or optimized filters, but in fact searchers may prefer different options or to be able to choose using a sliding scale of sensitivity depending on the number of records retrieved.

\section{Conclusion}

Search filters are used mainly for reducing large result sets (introducing focus) and assisting with searches which are focused on a single study type. Searchers use several key resources to identify search filters, but may find choosing between filters problematic. Features that would help with choices include making information about filters less technical, offering ratings, and providing more detail about filter validation strategies and filter provenance.

\section{Acknowledgements}

This research was funded as part of the UK Medical Research Council grant G0901496.

We would like to thank all the interviewees and questionnaire respondents for their time and their thoughtful and considered contributions. We are also grateful for the comments of the anonymous referees who reviewed this paper. 


\section{References}

1. Jenkins M. Evaluation of methodological search filters--a review. Health Info Libr J. 2004;21(3):148-63.

2. Jenkins M, Johnson F. Awareness, use and opinions of methodological search filters used for the retrieval of evidence-based medical literature-a questionnaire survey. Health Info Libr J. 2004;21(1):33-43.

3. The InterTASC Information Specialists' Sub-Group. The InterTASC Information Specialists' Sub-Group Search Filter Resource [Internet]. York: InterTASC Information Specialists' Sub-Group; 2014 [cited 2014 January 30]. Available from: https://sites.google.com/a/york.ac.uk/issg-search-filters-resource.

4. National Library of Medicine. PubMed Clinical Queries [Internet]. Maryland: National Library of Medicine; 2014 [cited 201430 January]. Available from: http://www.ncbi.nlm.nih.gov/pubmed/clinical.

5. White VJ, Glanville J, Lefebvre C, Sheldon TA. A statistical approach to designing search filters to find systematic reviews: objectivity enhances accuracy. J Inf Sci. 2001;27:35770.

6. Glanville JM, Lefebvre C, Miles JNV, Camosso-Stefinovic J. How to identify randomized controlled trials in MEDLINE: ten years on. J Med Libr Assoc. 2006;94:130-6.

7. Haynes RB, Wilczynski N, McKibbon KA, Walker CJ, Sinclair JC. Developing optimal search strategies for detecting clinically sound studies in MEDLINE. Journal of the American Medical Informatics Association. 1994;1(6):447-58.

8. Wilczynski NL, Morgan D, Haynes RB, Hedges T. An overview of the design and methods for retrieving high-quality studies for clinical care. BMC Med Inform Decis Mak. 2005;5:20.

9. Boynton J, Glanville J, McDaid D, Lefebvre C. Identifying systematic reviews in MEDLINE: developing an objective approach to search strategy design. J Inf Sci. 1998;24:137-54.

10. Bak G, Mierzwinski-Urban M, Fitzsimmons H, Morrison A, Maden-Jenkins M. A pragmatic critical appraisal instrument for search filters: introducing the CADTH CAI. Health Info Libr J. 2009;26(3):211-9.

11. Glanville J, Bayliss S, Booth A, Dundar Y, Fernandes H, Fleeman ND. So many filters, so little time: the development of a search filter appraisal checklist. J Med Libr Assoc. 2008;96:356-61.

12. Lefebvre C. Methodological search filter performance: assessment to improve efficiency of evidence information retrieval. MRC research grant G0901496. London: MRC2010.

13. Centre for Reviews and Dissemination. Centre for Reviews and Dissemination. York: Centre for Reviews and Dissemination; 2014 [cited 2014 January 28]; Available from: http://www.york.ac.uk/inst/crd/.

14. Health Information Research Unit. Hedges. Hamilton, On: McMaster University Health Information Rearch Unit; [cited 2014 January 28]; Available from: http://hiru.mcmaster.ca/hiru/HIRU_Hedges_home.aspx.

15. Wolters Kluwer. OvidSP. New York: Ovid Technologies; [cited 28 January 2014]; Available from: http://www.ovid.com/webapp/wcs/stores/servlet/topCategories?storeld=13051\&catalogld= 13151\&langld=-1.

16. National Library of Medicine. PubMed. Maryland: National Library of Medicine; [cited 201428 January]; Available from: http://www.ncbi.nlm.nih.gov/pubmed.

17. National Institute for Health and Care Excellence. Journals and Databases. London: National Institute for Health and Care Excellence; [cited 2014 January 28]; Available from: https://www.evidence.nhs.uk/about-evidence-services/journals-and-databases. 
18. Scottish Intercollegiate Guidelines Network. Scottish Intercollegiate Guidelines Network. Edinburgh: Healthcare Improvement Scotland; [cited 2014 January 28]; Available from: http://www.sign.ac.uk/index.html.

19. Hausner E, Waffenschmidt S, Kaiser T, Simon M. Routine development of objectively derived search strategies. Systematic Reviews. 2012;1:19.

20. Glanville J, Lefebvre C, Porter B, Negosanti P. Improving search efficiency for economic evaluations in major databases using semantic technology. The Cochrane Colloquium; Colorado, USA. October 2010. 


\section{Appendix}

\section{MRC SEARCH FILTERS PROJECT}

\section{Questionnaire}

1. Please state your job title:

2. How long have you been searching databases such as MEDLINE (years)?

3. How often do you develop new search strategies as part of your work (For example searches to find treatments for condition)?

None

Daily

Once a week

Once a month

Less than once a month

4. What types of searches do you carry out (please tick all that apply)

Rapid searches to answer brief queries

Scoping searches to estimate the size of the literature on a topic

Extensive searches to inform guidelines or systematic reviews

Other

If Other, please describe in the box below

5. Which databases do you search regularly?

MEDLINE

EMBASE

CINAHL

PSYCINFO

COCHRANE LIBRARY databases (CDSR, DARE, NHS EED, CENTRAL, HTA) HEED

Please list any other databases that you use regularly in the box below 
6. Methodological search filters (also known as Clinical Queries or Search Hedges) are used to find specific study designs such as randomised controlled trials. Have you ever used methodological search filters?

7. In what circumstances would you use methodological search filters?

Rapid searches to answer brief queries

Scoping searches to estimate the size of the literature on a topic

Extensive searches to inform guidelines or systematic reviews

Other

If Other, please describe below

8. Do you always use a filter when providing searches for similar types of projects? (For example, if you were searching for randomised controlled trials in MEDLINE would you always use a methodological search filter?)

If No, please provide details about the circumstances when you would not use a filter?

9. Please select the statement which describes your typical practice:

I use different search filters depending on whether my search has to be sensitive or precise

I use the same search filter irrespective of the focus of the search

10. If you had to find a methodological search filter for a specific study design, where would you look? 
11. What methodological search filters do you use at present?

Randomised controlled trials - please list the author or name of each of the filters you use?

Systematic reviews - please list the author or name of each of the filters you use

\begin{tabular}{|l|l|}
\hline \\
\hline
\end{tabular}

Diagnostic studies - please list the author or name of each of the filters you use

Studies of prognosis - please list the author or name of each of the filters you use

Studies of etiology - please list the author or name of each of the filters you use

Other trials - please list the author or name of each of the filters you use

Guidelines - please list the author or name of each of the filters you use 
Economic evaluations - please list the author or name of each of the filters you use

Other study methods - please list the author or name of each of the filters you use

12. How do you decide which filter to use? Please select all which apply

Custom and practice - l've always used the same filters

Guidance from a colleague

I research the available filters and chose the best for my purposes

I follow standing operating procedures/guidance on filters provided by my organisation

I use international/national guidance on best practice

I use the filters available in the database interfaces I use, e.g. Clinical Queries

Please provide details on any other approaches you use to decide which search filter to use

13. Apart from adding a subject search, do you amend methodological search filters?

No

Sometimes

Always

14. Please can you provide us with some more information about amending search filters?

Why, typically, do you amend search filters? 
How do you amend search filters?

Do you test the effects of any amendments you make?

Yes/No

If Yes, how do you test the amendments?

Do you document the amendments when you write-up your searches?

Yes/No

If Yes, how do you document the amendments?

15. How do you keep up to date with methodological search filters? (Please tick all the apply)

Reading journal articles

Current awareness services

Please list typical current awareness services that you use

Websites

Please list typical websites that you use

Professional development meetings and training events

Email lists

Please list typical email lists that you use 
RSS feeds

Please list typical RSS feeds that you use

Information provided by managers/work colleague

Please use the box below to describe any other methods you use to keep up to date with methodological search filters

16. If you have had to choose between methodological search filters what features or information has helped you to do so?

17. If you report your search process do you describe the filters you used?

Yes/No

18. If you report your search process do you justify your choice of filters used?

Yes/No

19. What do you think are the benefits of using methodological search filters?

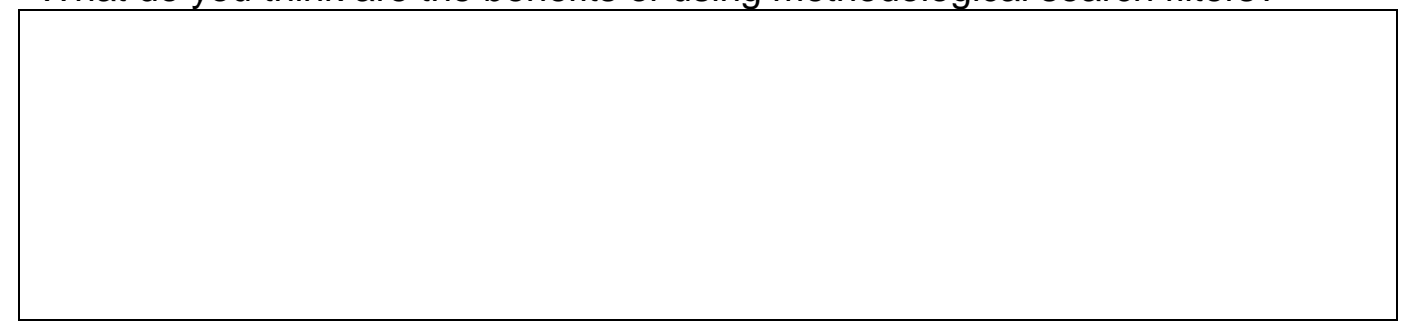

20. What do you think are the limitations of using methodological search filters? 
21. Imagine you have to choose between 2 or more methodological search filters:

What information would help you to choose which filter to use?

What would make choosing easier?

22. What methodological search filters would be useful to you?

W

23. Please use the box below to provide any further observations on methodological search filters as a tool for information retrieval

\section{Thank you for your help.}

